\title{
After the GT: Synthesizing Harrod's Knife-Edge Growth and Kaldor's Model of the Trade Cycle
}

\subsection{Harrod's knife-edge growth and Kaldor's trade cycle: Theory and empirics}

The Neo-Kaleckian model of growth and distribution is presently a popular workhorse in heterodox macroeconomics concerned with effective demand. There are nevertheless different problems arising for different camps of theorists. Underlying the present chapter is the view that in the reference position of a long-run equilibrium, even if it is never attained, the firms' actual utilization rate should coincide with an exogenous 'normal' rate of utilization. Methodologically, these economists are thus 'Keynesian in the short term and classical in the long term', to quote the telling title of Duménil and Lévy's (1999) well-known article. As these models typically include some sort of an accelerator mechanism, they face the problem of Harrodian instability.

Several remedies have been proposed in the literature to tame these centrifugal forces. ${ }^{1}$ Remaining within the sphere of pure quantity adjustments, Franke (2018a, 2019) recently sought to revive an old but practically forgotten idea by Harrod (1939) and Kaldor (1940). The argument is that even with identical expectations about the future, the history of their investments will still make a difference to the firms. That is, ceteris paribus current investment will be lower the higher the capacities they have already built up in the past, where it may be emphasized that the level of the existing capital stocks is assessed from the long-range perspective of the firms' normal market potential. When adding this feedback to Harrod's destabilizing 'acceleration principle', a curb is put on an ongoing expansion by the mere fact that the capital stock is continuously increasing in this phase. Therefore, investment and with it economic activity as a whole are bound to slow down and eventually turn around.

The interaction of just these two elementary forces, one stabilizing and the other destabilizing, can constitute the core of a cyclical dynamics. ${ }^{2}$ Franke (2018a) has worked out the specification details that permit an interpretation of this approach as a most fundamental business cycle theory. His model, which is called a Harrod-Kaldor business cycle model, can thus be characterized as going 
back to the beginnings of business cycle theory in the 1930s and 1940s and adapting their concepts to the now common growth framework of heterodox macroeconomics. ${ }^{3}$ In finer detail, the model also employs a modern tool to deal with the expectations of the firms, or better with a business climate. The general perspective is that by way of stepwise extensions such a highly (but sophisticatedly) simplified model can form a fruitful basis for a more comprehensive business cycle modeling in future work.

Franke (2018a) is a deterministic model formulated in continuous time which can be reduced to two dimensions. A convenient point of its canonical specification of the business sentiment adjustments is that it gives rise to an almost natural nonlinearity. This feature has the effect that near the model's unique steady state the trajectories are spiraling outward in the phase plane, while further away from it they are spiraling inward. In this way a unique and stable limit cycle comes into being. In order to interpret it as a business cycle, the chapter offers a rough-and-ready numerical calibration of the periodic orbit. That is, its period and the amplitudes of the model's observable variables are fitted to empirical data from the US.

Franke's model and its analysis are a promising first step, but under the perspective just mentioned one needs to be more ambitious. The present chapter therefore seeks to bring the numerical work to a higher level, for which it extends the original model slightly and then endeavours an econometric estimation. In this respect it should, however, be mentioned that here we will content ourselves with finding 'good' values for the numerical coefficients and only touch upon an assessment of the uncertainty or accuracy of the parameter estimates. As a matter of priority, we are rather primarily interested in the finer details of the model's goodness-of-fit, so that we obtain some systematic information about its merits and demerits. We believe it is only worthwhile to turn to parameter identification issues when we are largely satisfied with the possible 'realism' of the model. ${ }^{4}$

Technically speaking, the model is estimated by the method of simulated moments. This means a number of observable summary statistics, or 'moments', is selected and an objective function, or 'loss function', is minimized that measures the distance between the model-generated values and their empirical counterparts. The statistics themselves, which are concerned with a utilization rate and a so-called capital ratio, fall into two groups. The main group is given by a number of auto- and cross-covariances of the two variables. It may be noted that a good match of them also implies a similar average duration of the simulated and empirical cycles. This type of moments has already quite often been employed in applied work. ${ }^{5}$ The second group of moments is less conventional. They consider the different raggedness of the two variables, which the naked eye recognizes immediately and for which now a quantitative measure is provided.

A common criticism of the estimation approach concerns the choice of the moments. As there is no general rule for it, the method of simulated moments is accused of a certain arbitrariness. This argument should, however, be given a second thought, beginning with the basic question, 'what is a model?' Before 
anything else, a model by its very nature is an abstraction that seeks to isolate certain features of the mechanisms in the real world, while deliberately ignoring (abstracting from) the rest. After all, 'A model which took account of all the variegation of reality would be of no more use than a map at the scale of one to one' (Robinson, 1965, p. 33). ${ }^{6}$ Apparently, the objective function of the method of simulated moments is not something that is somehow able, or said to be able, to summarize all properties of a dynamic system. It rather summarizes a limited number of them. Thus it forces the researcher to disclose their priorities and make explicit the features that their model should try to reproduce as well as possible. Of course, criticism can be raised against a specific choice of moments, but not against the necessity of choosing them as such. In short, we may claim that the problem of the choice of moments is even a virtue of the estimation method rather than a vice.

The remainder of the chapter is organized as follows. Section 1.2 presents the model. For easier exposition, we first discuss its deterministic core in a continuous-time formulation. To prepare it for the estimation, it is subsequently transformed into discrete time and three shock process are added. Section 3 deals with the estimation. Section 1.3.1 recapitulates what we need to know about the moment matching approach for our purpose. Because they are of crucial importance, Section 1.3.2 has to defend our choice of the moments. Section 1.3 .3 presents the parameters of a reference scenario and gives a first impression of how close the (approximated) expected moments come to their empirical counterparts. Instead of the long simulation horizon in this section, Section 1.3.4 studies the small sample variability of the model. In particular, it proposes a concept that can measure a model's overall goodness-of-fit. Section 1.3.5 turns to the single moments and provides a measure of their matching performance. This allows a detailed moment diagnostic. Section 1.3.6 estimates several subversions of the model. It finds out that not all three shock processes are jointly needed for a good match, so that as a final upshot we can offer a slightly more parsimonious version of the model. Section 1.4 finally concludes.

\subsection{Harrod-Kaldor business cycle modelling}

The Harrod-Kaldor model is basically driven by the firms' expectations of future sales, which determine their investment in fixed capital and thus, via the multiplier effects, economic activity as a whole. In contrast to practically all lowdimensional business cycle models, however, the present model distinguishes between two time horizons in the investment decisions: a medium-term and a long-term perspective. The medium-term expectations centre around the familiar deviations of capacity utilization from normal, which over a wide range of specifications are well-known for a strong tendency to destabilize the economy.

The idea that long-term expectations may also play a role in the investment decisions can be traced back to Harrod (1939) and Kaldor (1940); see Franke (2019) for an elaboration of this point. Expectations of this kind are underlying the firms' evaluation of their stock of capital in place, whether it would turn out 
to be generally too high or too low in a more distant future. The benchmark for such a judgement is its ability to produce at decent levels of utilization the quantities that would satisfy what may be called 'potential' demand. This is a concept that, similar to the well-known concept of potential output, abstracts from the fluctuations at higher frequencies and seeks to capture a fundamental trend. The corresponding reactions in investment will be expected to have a stabilizing influence.

The two time horizons are reflected in the two key variables of the present proposal of a Harrod-Kaldor model. The first one represents medium-term expectations directly and corresponds to what is often called a business climate or sentiment, or more boldly 'animal spirits'. Alluding to the latter, we denote them by the symbol ' $a$ '. Specifically, the business climate will be a weighted average of optimistic and pessimistic growth expectations, where the weights and thus the climate itself change over time in response to the latest observations that the firms have made (specified later). The second variable is a composite expression $k$ that incorporates the long-term expectations. For short it is called a capital ratio. It replaces the level of the capital stock in, especially, Kaldor's model of a stationary economy and is defined as

$$
k=\frac{K}{K^{p}}=\frac{K}{D^{p} / u^{n}}
$$

While $K$ is the present capital stock of the firms, $K^{p}$ may be referred to as a normal or a potential stock of capital. We prefer the latter expression because it is based on the aforementioned concept of potential demand, $D^{p}$. In greater detail, $K^{p}$ would allow the production of $D^{p}$ at a normal rate of capital utilization (i.e. output-capital ratio) $u^{n}$, so that $D^{p}=u^{n} K^{p}$. The benchmark utilization itself is supposed to be exogenously given and fixed. As $k$ is specified, values less than unity, such that $K<K^{p}$, indicate that $K$ tends to be too low in a long-term perspective. In this case the firms will increase their fixed capital at a somewhat higher rate than the one determined by the other investment motives. Vice versa when $k>1$. Actually, as discussed in Franke (2019), this is the idea of what Harrod (1939, pp. 26ff) called the 'long-range capital outlay'.

We are thus ready to put forward our function of the (net) investment expenditures. Together with the usual reactions of investment to changes in the difference between actual and normal output-capital ratios, $u-u^{n}$, it reads:

$$
g^{i}=a+\gamma_{u}\left(u-u^{n}\right)-\gamma_{k}(k-1)
$$

where $g^{i}$ are the expenditures as a ratio of the capital stock, $\gamma_{u}$ and $\gamma_{k}$ are two constant nonnegative reaction coefficients, and $k-1=\left(K-K^{n}\right) / K^{n}$ are the percentage deviations of the capital stock from its potential level. Clearly, regarding the expectations that are underlying this investment rule, the rate $a$ together with the utilization gap $u-u^{n}$ represents the medium-term perspective, and $(k-1)$ the long-term perspective. Intuitively, the first may be conducive to a positive feedback loop, Harrod's (1939, p. 14) instability or 'acceleration principle' in fact, and the second to a negative feedback loop. The precise conditions for them to come about are analyzed in Franke (2018a, 2019). 


\subsubsection{The deterministic model}

As in so many macro models, the framework is that of a closed one-good economy where labour is supposed to be in perfectly elastic supply. The measure of overall economic activity is the output-capital ratio $u=Y / K$ ( $Y$ being total output), which conforming to the introduction of $u^{n}$ will also be referred to as (capital) utilization. It is determined in a familiar IS manner, that is, its variations are assumed to continuously clear the goods market. To this end the other components of demand are kept particularly simple, though we slightly complicate the notation by introducing two specification details that will allow us to limit the otherwise excessively strong multiplier effects.

Neglecting depreciation, the profit rate $r$ is the product of utilization $u$ and the share of profits in total income, $h$. Again for simplicity, we abstain from possible effects of the variations in income distribution and treat $h$ as a fixed parameter. Wage and profit income is taxed at a uniform rate $\tau$. After-tax real wages, which are given by $(1-\tau)(1-h) Y=(1-\tau)(1-h) u K$, are entirely spent on consumption. The consumption of the rentier households, who own the firms, is based on two components: first, on their current after-tax dividend income $(1-\tau) h u K$, to which a marginal propensity to consume $(1-s)$ is applied (there are no retained earnings of firms); and second, with a proportionality factor $\tilde{c}_{o}$, on what the rentiers perceive as their permanent income, that is, the 'normal' real profits $(1-\tau) r^{n} K:=(1-\tau) h u^{n} K$. Putting $c_{o}:=\tilde{c}_{o}(1-\tau) h u^{n}$, total consumption amounts to $(1-\tau)(1-h) u K+(1-s)(1-\tau) h u K+c_{o} K$.

Government spending $G$ is given by a simple rule with a certain countercyclical component. It is supposed to be proportional with a factor $\tilde{c}_{G}$ to normal national income $Y^{n}:=u^{n} K$, so that with $c_{G}:=\tilde{c}_{G} u^{n}, G=\tilde{c}_{G} Y^{n}=c_{G} K{ }^{7}$ The clearing condition for the goods market is given by the equality of output on the one hand, and investment plus consumption plus government spending on the other hand. Dividing this equation by the capital stock, we obtain the relationship

$$
u=g^{i}+(1-\tau)(1-h) u+(1-\tau)(1-s) h u+c_{o}+c_{G}
$$

Substituting the investment function 1.2 and solving $(1.3)$ for $u$, IS utilization is given by

$$
\begin{aligned}
u=u(a, k) & =\left(a-\gamma_{k} k+C\right) / D \\
C & :=c_{o}+c_{G}-\gamma_{u} u^{n}+\gamma_{k} \\
D & :=\tau+(1-\tau) s h-\gamma_{u}
\end{aligned}
$$

The Keynesian stability condition requires $D>0$. It is the merit of the extension $c_{o}>0$ in the standard IS formulation that it makes a higher marginal savings propensity $s$ possible, which raises the denominator $D$. Positive tax rates $\tau$ can increase it even further. The multiplier effects, which in the presence of $c_{o}=\tau=0$ would typically be unreasonably strong, can thus be considerably moderated. To what realistic extent, will have to be seen when it comes to the numerical issues of the model. 
With respect to a pair $(a, k)$, investment expenditures $g^{i}$ in the corresponding IS equilibrium are given by

$$
g^{i}=g^{i}(a, k)=a+\gamma_{u}\left[u(a, k)-u^{n}\right]-\gamma_{k}(k-1)
$$

In the great majority of macro-dynamic modelling, these outlays are instantaneously transformed into the intended change in capacity. Accordingly, the actual capital growth rate coincides with $g^{i}$ and therefore moves synchronically with utilization $u .^{8}$ We may anticipate here that in the deterministic cycles of $u$ and $k$ that we are interested in, there would then be a phase shift of these two variables of exactly a quarter of a cycle. This feature implies a zero correlation between the contemporaneous values of $u$ and $k$. Empirically, however, the correlation coefficient is significantly negative, which is caused by the regularity that the capital growth rate lags behind utilization by two or three quarters.

For this reason we drop the otherwise convenient simplification $g=g^{i}$, where $g$ denotes the actual growth rate of the capital stock. One way to do this is to follow Kalecki's (1935) business cycle model (or its adaptation to a growth context by Franke (2018c)) and introduce a so-called gestation period of investment. In a continuous-time framework this would lead to a delaydifferential equation, something that is not only less familiar to many economists but also much harder to analyze. ${ }^{9}$ Here we resort to a simpler device which treats $g$ as a dynamic variable that adjusts toward $g^{i}$ with some delay. To ease the exposition and facilitate a comparison with Franke (2018a), this and the other dynamic relationships are provisionally formulated in continuous time. Thus, with a speed of adjustment $\alpha>0$, the changes of the capital growth rate are described by the ordinary differential equation, ${ }^{10}$

$$
\dot{g}=\alpha\left[g^{i}(a, k)-g\right]
$$

Note that in the limiting case where $\alpha$ tends to infinity, we would be back in the ordinary treatment $g \equiv g^{i}$. On the other hand, lowering the coefficient $\alpha$ increases the lag between $g$ and $g^{i}$ and thus between $g$ and $u$.

Besides the capital growth rate, the changes in the capital ratio $k$ are determined by the firms' projections of the path of potential demand $D^{p}$. Its basic element is the evolution of the number of future customers and the trend of their purchasing power. In growth terms this is the growth rate of the labour force and the rate of technical progress. In the deterministic model we neglect any variability in this kind of expectations and treat the two growth rates as exogenously fixed magnitudes. Their sum constitutes the natural rate of growth, $g^{n}$. Accordingly, the projected growth rate $g^{p}$ of potential demand reads,

$$
g^{p}=\dot{D}^{p} / D^{p}=g^{n}
$$

By logarithmic differentiation of the capital ratio, $\hat{k}=\hat{K}-\hat{D}^{p}$, which gives us

$$
\dot{k}=k\left(g-g^{n}\right)
$$


To specify the motions of the business climate $a$, we make use of the recent literature on a rigorous modelling of 'animal spirits'; see Franke and Westerhoff (2017) for a survey. Let us assume that the individual firms can have either optimistic or pessimistic growth expectations for the medium term. ${ }^{11}$ Denoting these expected growth rates by $a^{+}$and $a^{-}$, respectively, and the corresponding shares of optimistic and pessimistic firms by $n^{+}$and $n^{-}$, the average expected growth rate is $a=n^{+} a^{+}+n^{-} a^{-}$. It is convenient to set $a^{+}=g^{n}+1 / \xi$ and $a^{-}=g^{n}-1 / \xi$ for some $\xi>0$ and to refer to a majority index $x:=n^{+}-n^{-}$. It will be useful that in this way expected growth, or the business climate, is directly linked to the index $x$,

$$
a=g^{n}+(1 / \xi) x
$$

Certainly, $x$ is contained between plus and minus one, and $a$ between $a^{+}$ and $a^{-}$. The crucial point is that $x$ varies over time. The updating process is specified by the established discrete choice approach. However, contrary to many applications of this tool in the literature, firms are not required to reconsider their attitude synchronously every month or quarter. ${ }^{12}$ We rather assume that a single firm only does this with a fixed probability $\mu>0$ per unit of time. Then, when it does, it chooses to be optimistic and pessimistic with certain probabilities of the discrete choice type. They are not constant but functions of a so-called switching index $z$, which in turn will be a function of the macro variables $u$ and $k$. The convention is that higher values of $z$ are conducive to more optimism in the business sector. Conducting some elementary mathematical manipulations, the changes in the majority index can without loss of generality be shown to be described by

$$
\dot{x}=\mu[\tanh (z)-x]
$$

see, e.g., Franke (2018a, p. 6) or Franke and Westerhoff (2017, Section 2.1) for the finer details. ${ }^{13}$ The idea is that firms become more optimistic when the utilization rate $u$ rises and more pessimistic when the capital ratio $k$ rises. In addition a herding component is allowed for. It says that a higher share of optimistic firms (a higher value of $x$ ) increases the probability of all firms to become optimistic; and reversely for a higher share of pessimistic firms. These three effects are represented by the switching index $z$, which in detail is specified as a linear function of the three variables. Knowing already the solution (1.4) for IS utilization $u=u(a, k)$ with its linearity, $z$ can be expressed as a linear function of $(x, a, k)$ :

$$
z=z(x, a, k)=\phi_{x} x+\phi_{u}\left[u(a, k)-u^{n}\right]-\phi_{k}(k-1)
$$

The three reaction coefficients $\phi_{x}, \phi_{u}, \phi_{k}$ are nonnegative, of course. The majority index $x$ can be eliminated from the model by expressing it in terms of the climate variable $a$. First, solving eq. 1.9 for $x$ and substituting it in (1.11) gives us

$$
z=z(a, k)=\phi_{x} \xi\left(a-g^{n}\right)+\phi_{u}\left[u(a, k)-u^{n}\right]-\phi_{k}(k-1)
$$

Subsequently, with $\dot{a}=(1 / \xi) \dot{x}$ from 1.9$)$ for the time derivative, eq. 1.10$)$ can be rewritten as 


$$
\dot{a}=(\mu / \xi)\left\{\tanh [z(a, k)]-\xi\left(a-g^{n}\right)\right\}
$$

This completes the formulation of the deterministic Harrod-Kaldor model. In sum, it is a three-dimensional differential equation system for the motions of $a, k$ and $g$, constituted by equations $(1.13),(1.8),(1.6)$ together with the expressions for utilization, investment expenditures and the switching index in equations (1.4), 1.5, 1.12, respectively.

The model reduces to the two-dimensional system studied in Franke (2018a) if we let the speed of adjustment $\alpha$ in 1.6 tend to infinity. For finite values of $\alpha$, on the other hand, it is easily demonstrated that the original condition for the local stability of the steady state position, which there was necessary and sufficient, is preserved as a necessary condition. To guarantee stability, however, it has to be combined with two conditions on $\alpha$ that say this coefficient must not be too low. Because of our interest in the model's persistent cyclical behaviour and its numerical features, which (as it turns out) requires the steady state to be unstable, we omit the details of a mathematical stability proposition.

\subsubsection{The quarterly stochastic model}

For an empirical investigation it is convenient to transform the continuous-time formulation into discrete time. To this end time is sliced into periods of a quarter of year. This means the year is maintained as the time unit and a quarter is the length of the adjustment period. Accordingly all flows are expressed as annualized magnitudes, while the dynamic variables are considered in periods

$$
t \in Q:=\{0, q, 2 q, 3 q, \ldots\}, \quad \text { where } q:=0.25
$$

In this setting also random shocks can be introduced in a straightforward way. They may serve as a proxy for some additional and nonnegligible forces that, however, are not explained within the present model. Although they can enrich the model and its dynamic properties, one should not be too liberal in this respect. We thus consider three obvious stochastic process and let the estimations later on decide whether they are helpful or whether some of them may be dispensable. To account for a certain shock persistence, all of them are specified as first-order autoregressive processes.

The first source of noise in the model seeks to capture the vagaries in the components of aggregate demand. This is conveniently specified by adding an autoregressive term $\eta_{u}$ on the right-hand side of the market equilibrium equation 1.3. IS utilization in period $t$ together with the corresponding investment expenditures are then given by $(C$ and $D$ as defined in (1.4)):

$$
\begin{aligned}
u_{t}=u\left(a_{t}, k_{t}, \eta_{u, t}\right) & =\left(a_{t}+\eta_{u, t}-\gamma_{k} k+C\right) / D \\
\eta_{u, t} & =\rho_{u} \eta_{u, t-q}+\varepsilon_{u, t}, \quad \varepsilon_{u, t} \sim N\left(0, \sigma_{u}^{2}\right) \\
g_{t}^{i}=g^{i}\left(a_{t}, k_{t}, \eta_{u, t}\right) & =a_{t}+\gamma_{u}\left[u\left(a_{t}, k_{t}, \eta_{u, t}\right)-u^{n}\right]-\gamma_{k}\left(k_{t}-1\right)
\end{aligned}
$$

It goes without saying that the autocorrelation coefficient $\rho_{u}$ is contained between zero and one, and the normally distributed innovations $\varepsilon_{u, t}$ are i.i.d. with standard deviation $\sigma_{u}$. Analogously for the shock processes below. 
The other two random shocks act on the medium- and long-term expectations. One is attached to the switching index that governs the adjustments of the business climate, the other to the firms' projections of the growth of potential demand. Regarding the switching index, the perturbations can be directly added to the function $z=z(a, k)$ from $(1.12$. It now becomes,

$$
\begin{aligned}
z_{t} & =z\left(a_{t}, k_{t}, \eta_{u, t}, \eta_{z, t}\right) \\
& =\phi_{x} \xi\left(a_{t}-g^{n}\right)+\phi_{u}\left[u\left(a_{t}, k_{t}, \eta_{u, t},\right)-u^{n}\right]-\phi_{k}\left(k_{t}-1\right)+\eta_{z, t} \\
\eta_{z, t} & =\rho_{z} \eta_{z, t-q}+\varepsilon_{z, t}, \quad \varepsilon_{z, t} \sim N\left(0, \sigma_{z}^{2}\right)
\end{aligned}
$$

Regarding the long-term expectations, we drop the constant growth rate $g^{p}$ for the projections of potential demand and admit some exogenous variations. The natural growth rate $g^{n}$ as an anchor is nevertheless maintained:

$$
\begin{aligned}
g_{t}^{p} & =g^{n}+\eta_{p, t} \\
\eta_{p, t} & =\rho_{p} \eta_{p, t-q}+\varepsilon_{p, t}, \quad \varepsilon_{p, t} \sim N\left(0, \sigma_{p}^{2}\right)
\end{aligned}
$$

The discrete-time analogues of the changes in the business climate and the capital growth rate in equations 1.13 and (1.6), respectively, are straightforward:

$$
\begin{aligned}
& a_{t+q}=a_{t}+(q \mu / \xi)\left\{\tanh \left[z\left(a_{t}, k_{t}, \eta_{u, t}, \eta_{z, t}\right)\right]-\xi\left(a_{t}-g^{n}\right)\right\} \\
& g_{t+q}=g_{t}+q \alpha\left[g^{i}\left(a_{t}, k_{t}, \eta_{u, t}\right)-g_{t}\right]
\end{aligned}
$$

With respect to the changes in the third state variable $k_{t}$ we should be less mechanical. Since it is a composite expression, the discrete-time changes of its components $K_{t}$ and $D_{t}^{p}$ should be considered separately. Here we have $K_{t+q}=$ $\left(1+q g_{t}\right) K_{t}$ and, from $1.20, D_{t+q}^{p}=\left(1+q g_{t}^{p}\right) D_{t}^{p}=\left[1+q\left(g^{n}+\eta_{p, t}\right)\right] D_{t}^{p}$, so that $k_{t+q}=K_{t+q} /\left(D_{t+q}^{p} / u^{n}\right)=\left(1+q g_{t}\right) K_{t} /\left\{\left[1+q\left(g^{n}+\eta_{p, t}\right)\right] D_{t}^{p} / u^{n}\right\}$. This gives us

$$
k_{t+q}=\frac{1+q g_{t}}{1+q\left(g^{n}+\eta_{p, t}\right)} k_{t}
$$

It is readily checked that if, for the sake of the argument, $\eta_{p, t}=0$ and $q \rightarrow 0$, (1.24 results in the differential equation 1.8 from above.

The description of the stochastic Harrod-Kaldor model is thus complete, such that it is ready for estimation. Inserting the expressions for $g^{i}\left(a_{t}, k_{t}, \eta_{u, t}\right)$, $z\left(a_{t}, k_{t}, \eta_{u, t}, \eta_{z, t}\right)$ and choosing initial conditions for the state variables $a, g, k$ as well as for the shock variables $\eta_{u}, \eta_{z}, \eta_{g}$, the simulations of the model can be carried out by simple forward iterations of the three dynamic key equations (1.22), 1.23), 1.24) and the three autoregressive processes (1.16), (1.19), (1.21).

\subsection{Estimation of the model}

\subsubsection{The estimation approach}

The method of simulated moments deals with a set of empirical summary statistics, or 'moments', that the model should try to match as closely as 
possible. Most commonly (but not necessarily) if the observed variables are stationary, moments are time averages of functional expressions involving their values in period $t$ and possibly some lags. These expressions may also be called moment functions. To simplify the description, let $t$ in the present context be integer numbers and $T$ the effective length of the sample period, which means that with $L$ the largest lag, data are available for $t=-(L-$ 1), $\ldots, 0,1, \ldots, T$. Let $y_{t}^{e m p}$ be a vector of observations in period $t$ and write $z_{t}^{e m p}=\left(y_{t}^{e m p}, y_{t-1}^{e m p}, \ldots, y_{t-L}^{e m p}\right)$. In total, $i=1,2, \ldots, n_{m}$ moment functions $m_{i}=m_{i}\left(z_{t}\right)$ are considered, where generally $z_{t}$ may represent empirical or simulated data. The empirical moments are thus given by

$$
m_{T, i}^{e m p}=(1 / T) \sum_{t=1}^{T} m_{i}\left(z_{t}^{e m p}\right), \quad i=1,2, \ldots, n_{m}
$$

For greater clarity, the index $T$ makes the sample period explicit.

Turning to our theoretical model, let there be $n_{p}$ parameters to be estimated and collect them in a vector called $\theta \in \mathbb{R}^{n_{p}}$. Given numerical values for its components, the model is run over an extended period of time and a sufficiently long initial history is discarded to wash out possible transient effects. In effect, we remain with $S$ model-generated observations $\left\{z_{t}(\theta)\right\}_{t=1}^{S}$, where $\theta$ refers to the underlying parameter vector. Occasionally we also make a random seed $c$ for the stochastic dynamics explicit, $z_{t}=z_{t}(\theta ; c)$ ( $c$ may conveniently be thought of as an integer number $)$. The vector $m=\left(m_{1}, m_{2}, \ldots, m_{n_{m}}\right)$ of the simulated moments thus reads,

$$
m_{S}(\theta)=m_{S}(\theta ; c)=(1 / S) \sum_{t=1}^{S} m\left[z_{t}(\theta ; c)\right]
$$

Supposing the stochastic system is ergodic so that the probability distribution of the variables converges to a unique invariant distribution, 1.26 approximates the correspondingly expected moment values if $S$ is sufficiently large. ${ }^{14}$ It is these expected moments that the estimation is concerned with. Certainly, it aims to bring them as close as possible to the empirical moments.

To this end the squared deviations of the simulated from the empirical moments are considered. As the moment statistics may be of a different order of magnitude, they have to be scaled to be on an equal footing. The interrelationships between the single moments can be accounted for by employing an $n_{m} \times n_{m}$ weighting matrix and minimizing the corresponding quadratic form of the vector of the moment deviations. There is furthermore a textbook recipe for setting up an optimal matrix for this function. It is, however, not a mandatory procedure. First, the matrix is only asymptotically optimal, whereas we have to deal with rather few observations and not much is known about the small sample properties of the estimation under these circumstances. Second, the matrix is optimal in reducing the uncertainty about the estimated parameters, which is not our main interest. Third, an optimal weighting matrix need not necessarily produce the closest match of the moments. Franke (2009) is 
an example which shows that a diagonal matrix (especially when combined with another naive modification of the objective function) may yield better results in this respect.

Because the focus in this chapter is not so much on parameter inference but on the matching potential of the model, we do not follow the pure econometric theory and content ourselves with a diagonal weighting matrix, that is, we just attach weights $w_{i}>0$ (explicated in a moment) to the squared deviations of moment $i$. In some cases we even deviate from the simple rule determining the $w_{i}$, in order to give these moments a higher priority in the matching procedure. The weighted squared deviations are then added up and, settling down on a fixed random seed $c=\bar{c}$, a numerical algorithm searches for a parameter vector that minimizes this expression:

$$
J_{S}(\theta ; c):=\sum_{i=1}^{n_{m}} w_{i}\left[m_{S, i}(\theta ; c)-m_{T, i}^{e m p}\right]^{2}=\min _{\theta} ! \quad(c=\bar{c} \text { fixed })
$$

An objective function of this type is often called a loss function. It is an obvious idea that moments which are measured with higher precision are given greater importance in the loss function. Accordingly, the $w_{i}$ are determined as the reciprocal of the variance of the empirical moments, i.e. their squared standard errors. A common recipe to compute them is an application of the delta method (cf. Davidson and MacKinnon 2004, pp. 207ff for example). To better account for the small empirical sample that we have, we prefer a bootstrap procedure. Fortunately the block bootstrap with its frequent breaks in the dependency structure can be avoided. Instead, with respect to a specific random seed $b \in$ $\mathbb{N}$, we sample (with replacement) from the index set $\{1,2, \ldots, T\}$ to obtain another set of indices $I(b)=\left\{t_{1}(b), t_{2}(b), \ldots, t_{T}(b)\right\}$, and then construct the moment vector $m_{T}^{b}$ as

$$
m_{T}^{b}=\sum_{t_{k} \in I(b)} m\left(z_{t_{k}}^{e m p}\right)
$$

Note that some periods will show up several times in the sum and others not at all. Repeating this $B$ times, for each component $i$ there is a collection of moments $\left\{m_{i}^{b}\right\}_{b=1}^{B}$, where the required standard error $\mathrm{SE}_{i}$ is just their standard deviation. Practically, $B=5000$ is large enough. In this way we also get a confidence interval $C_{i}$ for each moment in which we would like the simulated moments to lie: conventionally, $C_{i}=m_{T, i}^{e m p} \pm 2 \cdot \mathrm{SE}_{i}$ (in loose notation). Strictly speaking, the intervals should be a yardstick for simulations over the shorter empirical time horizon $T$, but this issue is taken up further below.

We wish to emphasize that the width of the bootstrapped confidence intervals is on average about 60 per cent of what one derives from the delta method. This means our standard for what we consider to be a good fit is relatively demanding.

\subsubsection{Selection of the moments}

A major part of a model's dynamic properties can be described by the autocovariances of its variables at a larger (but of course finite) number of 
quarterly lags. We turn to them in a moment. Before another and often neglected feature should be mentioned that is not directly captured by these statistics, although it immediately leaps to the naked eye if we look at a time series. This is their raggedness. Especially if it differs between two variables, we can make a quick qualitative assessment that one is not at all, or weakly, or strongly more ragged than the other.

Certainly, for a formal treatment an unambiguous summary statistic is needed. To this end, let us begin with a straightforward description which would call a single time series extremely ragged if it, from one observation to another, continuously alternates between increasing and decreasing. It appears 'fairly' ragged if this occurs rather frequently, and the series is almost perfectly smooth if it steadily increases or decreases except for some infrequent turning points. Furthermore, a time series where, for example, a strong increase between two observations is followed by a weak decline is, at that stage, less ragged than a series where the opposite changes are of a similar order of magnitude. These verbal classifications of what our eye perceives without further reflection lead us to measure the raggedness of a time series $\left\{x_{t}\right\}_{t=1}^{N}(N=T, S)$ in the following way, ${ }^{15}$

$$
R_{N}\left(x_{t}\right):=\frac{\sum_{t=3}^{N} \max \left[0,-\left(x_{t}-x_{t-1}\right)\left(x_{t-1}-x_{t-2}\right)\right]}{\sum_{t=3}^{N}\left|\left(x_{t}-x_{t-1}\right)\left(x_{t-1}-x_{t-2}\right)\right|}
$$

This statistic is independent of the length and scale of the time series and can vary between unity and zero, indicating perfect raggedness and perfect smoothness, respectively. The index $N$ for the sample period $T$ or the simulation horizon $S$ can be omitted in the following discussion since it will be easily understood from the context.

The variables entering our estimations are the output-capital ratio $u$ (or utilization) and the capital ratio $k$. Their empirical values refer to the nonfinancial corporate business sector of the US economy over the period 1960:q1-2007:q2, just before the financial crisis began to spread over to the real economy. Thus, $T=47.50$ years $=190$ quarters. The empirical capital ratio is obtained as the percentage deviations of the capital stock from its Hodrick-Prescott trend with the usual smoothing parameter $\lambda=1600$ for quarterly data, the output-capital ratio is detrended in the same way. The trend itself is computed over a longer period of time in order to avoid the end-of-period effects. ${ }^{16}$ The two time series are plotted in figure $1.1{ }^{17}$ Their different raggedness is not dramatic (as it is in the case of output and inflation rates) but still clearly visible. For $u$ it is quantified as $\mathrm{Ru}=0.2588$, for $k$ as $\mathrm{Rk}$ $=0.0187$.

Another summary statistic that so far has not been employed in moment matching estimations is a measure of stochastic volatility, or so-called fat tails. De Grauwe (2012) argues that excess kurtosis of quarterly output growth rates (and inflation) is a basic empirical fact, and he is proud that his behavioural New-Keynesian model (in contrast to the standard New-Keynesian model) can generate it to some degree, even though the shocks in his model are normally distributed. Jump and Levine (2019) follow him in this respect. We have two 

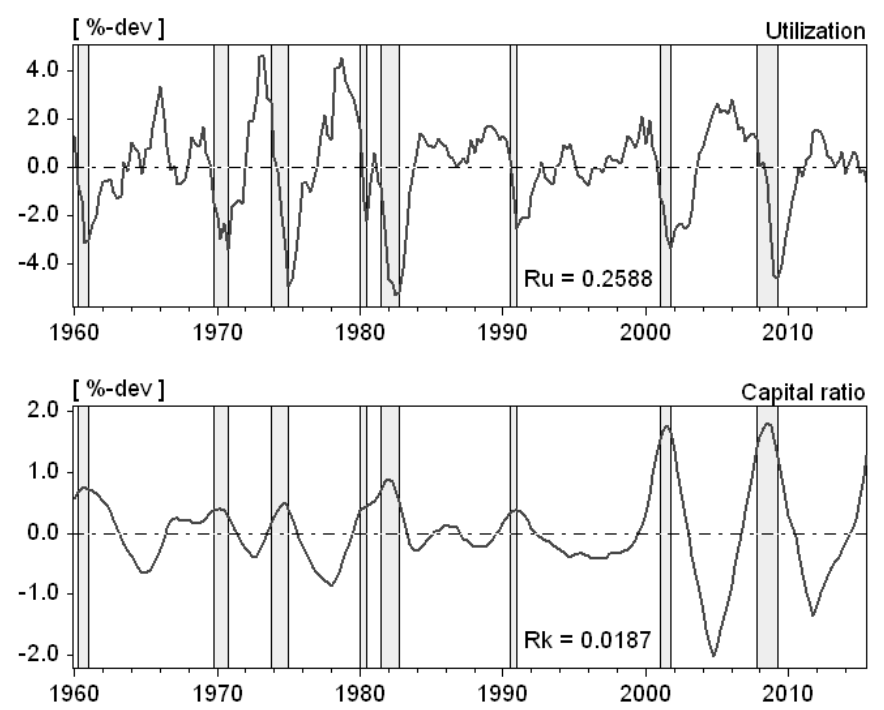

Figure 1.1: Trend deviations of the output-capital ratio $u$ and the capital ratio $k$.

critical remarks on this idea. First, kurtosis is not a very reliable measure of fat tails because it is very sensitive to outliers. A better approach is to assume that the output growth rates follow the exponential power distribution and estimate its shape parameter $b$ (the exponent): for $b=2$ the normal distribution is obtained, while values near unity, or at least significantly lower than 2 , are an indicator of fat tails. There are indeed several studies confirming the latter. Ascari et al. (2015) even raise $b \ll 2$ into the category of a stylized fact, i.e., a standard that ambitious macroeconomic models should seek to meet.

Second, referring to this conceptual framework, the evidence provided in these studies is less clear than it seems. A problem is the neglect of a structural break in the volatility of the growth rates over the relatively long sample periods they typically employ. In a stylized Monte Carlo experiment, Franke (2015) points out that the estimated fat tails over the entire period may also be explained by two pasted regimes of normality, but different average growth. In his subsequent estimations he found that whether or not fat tails prevail heavily depends on the kind of output data considered and the sample period over which the estimations are carried out (the Great Inflation or the Great Moderation, respectively). In short, evidence of fat tails is presently still rather mixed. We therefore abstained from considering the shape parameter $b$ as a moment of its own. ${ }^{18}$

We can thus turn to our main moments, which are the autocovariances (ACVs) of the two variables $u$ and $k$. They contain similar information to impulseresponse functions, which also serve to reveal the 'stylized facts' of the economy. To introduce a shorthand notation, let us, with respect to two dynamic variables $\left\{x_{t}\right\},\left\{y_{t}\right\}$ and a lag of $\ell$ quarters, abbreviate the covariance $\operatorname{Cov}\left(x_{t}, y_{t-q \ell}\right)$ by 
Table 1.1: The moments to match.

\begin{aligned} & \hline acronym moment description \\ & \hline uu0 variability (amplitude) \\ & kk0 0 phase shift $k$ vs. $u \\ &$. uu6 cycle period \\ & kk7 \\ & uk11 first zero correlation \\ & ku3 \\ & ku17 late zero correlation \\ & uu12 medium-term persistence \\ & $\mathrm{k} k 14 \\ & \mathrm{uk} 17 \\ & \mathrm{ku} 9 \\ & \mathrm{Ru} \\ & \mathrm{Rk} \\ &$\hline\end{aligned}

'xy $\ell$ '. Certainly, $x$ and $y$ can stand for $u$ and/or $k$. It has to be admitted that the model has only a limited capability to reproduce the statistics of other variables. ${ }^{19}$ The covariances that we select for the estimation together with the two raggedness moments $\mathrm{Ru}$ and $\mathrm{Rk}$ are listed in table 1.1. In sum, there are 14 moments that we want the model to approximate. Figure 1.2 shows the profile of all autocovariances of $u$ and $k$ up to a lag of 20 quarters, where the dots indicate the moments chosen in the table. The shaded area is the confidence band, which was bootstrapped as described in the previous subsection.

It is obvious that the variances (or standard deviations) of the variables have to be included in a moment estimation. Regarding the cross-relationship between $u$ and $k$, the two variables seem to be nearly inversely related when we look at the time series plot in figure 1.1. It should nevertheless not be neglected that the peaks of $k$ show a short lead relative to the troughs of $u$, so there is a small phase shift. It is, however, different from the pattern that one gets from the original model in Franke (2018a) where, corresponding to setting $\alpha=\infty$ in our equation (1.6), investment $g^{i}$ and the capital growth rate $g$ coincide. This version of the model systematically exhibits a phase shift of the two variables of about a quarter of a cycle, which was our reason for introducing a positive delay in (1.6), i.e. a finite adjustment speed $\alpha$. Whereas a quarter-of-a-cycle phase shift would be reflected by a zero cross-covariance uk0, the significantly negative value of this coefficient in figure 1.2 indicates a different timing in the comovements. ${ }^{20}$ These observations may explain why uk0 is introduced as a phase shift moment in table 1.1 . 

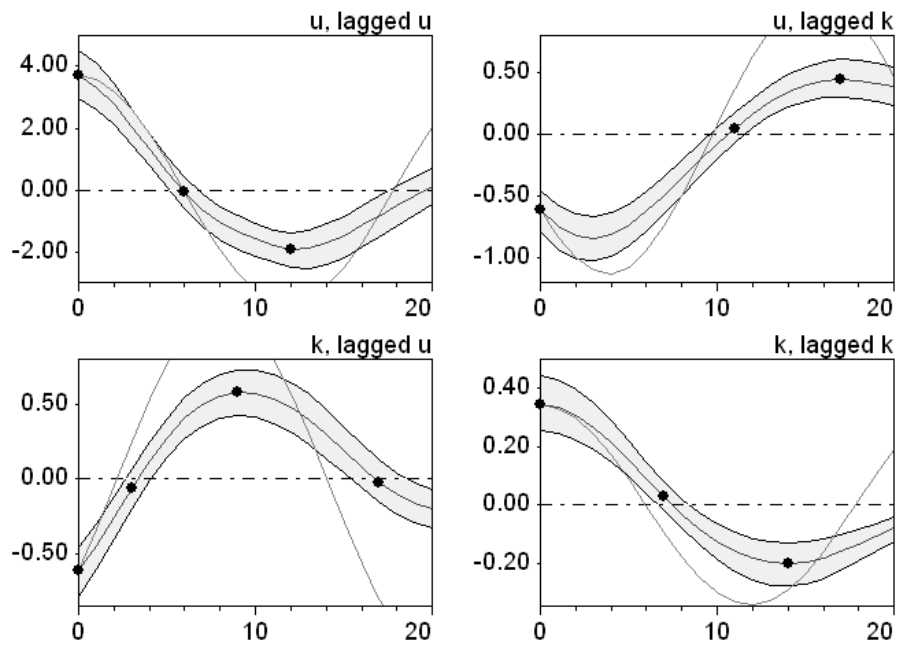

Figure 1.2: The empirical autocovariances and their confidence band.

The next two covariances uu6 and $\mathrm{kk} 7$ are motivated by the desire that the model reproduces the average length of a business cycle. Note in this respect that the period $P$ of a regular deterministic motion of a variable like a periodic sine wave is reflected in a profile of its $\mathrm{ACVs}$ that repeats itself after $P$ years, when it reaches its next peak. Its trough (as a mirror image of its peak) is obtained after $P / 2$ years, and after about $P / 4$ years the ACV will be zero. So we look for the nearest quarter when the profile of the ACVs cuts the zero line. For the empirical utilization rate $u$ this is the case at a lag of 6 quarters, for the capital ratio $k$ it is a lag of 7 quarters. Accordingly, without identifying specific peak and trough quarters of the variables or invoking a spectral analysis, figure 1.2 indicates an average period somewhat longer than 7 years ( 7 rather than 6 because $k$ is a smoother series than $u$ ).

The deterministic limit cycle in the simple Harrod-Kaldor model in Franke (2018a) was directly geared to the empirical amplitudes of $u$ and $k$, to the lag between $u$ and $k$, and to an intuitive choice of the cycle period. With respect to the present ACV profile, this corresponds to an exact match of the moments uu0, kk0, uk0, and either uu6 or kk7. Although with the adjustment speed $\alpha$ for the capital growth rate in $(1.6)$ the present model has an extra parameter, it was not possible to match in addition one of the other moments in table 1.1. The thin solid (red) line in figure 1.2 shows the best that the deterministic version of the model could achieve. Together with a short comment, the numerical parameters bringing it about are given below.

The role of the zero ACVs inspired us also to consider the cross-covariances of $u$ and $k$ at those quarters where they are first approximately zero, which explains uk11 and ku3 in table 1.1 . In our initial explorations of the matching procedure 
it turned out that ku3 was not too difficult to reproduce to a satisfactory degree, but the next ACV of these variables close to zero at lag 17 was often missed by larger margins. Since occasionally and taken by itself the match happened to be not too bad, either, moment ku17 was added as a further challenge to the model.

The persistence of a variable is commonly measured by its first-order autocorrelation. When ACVs at lag 1 were employed in the loss function, a good match of them tended to be implied by good matches of the other moments. In this sense their information content was only limited, so that we decided to discard them again. As a less 'local' indicator of a variable's persistence one could consider the difference between its highest and lowest ACV, an idea that may be called medium-tern persistence. Accordingly, with respect to utilization which attains its highest and lowest $\mathrm{ACV}$ at lag 0 and 12, respectively, we included uu12 as an additional moment. Similarly so for kk14, uk17, ku9, the late troughs or peaks in their ACV profile.

As already indicated, autocovariances have also been underlying other macroeconomic moment matching estimations, although at shorter lags only. The largest lag $L$ that we know of is $L=8$ (Franke, 2018b, Jang and Sacht, 2016, 2019) The fact that more than a half of the AVCs in table 1.1 are based on longer lags shows that the present moment selection is a rather ambitious one. It may also be noted that the 14 moments are largely independent of each other.

\subsubsection{Parameter estimates and the model's expected moments}

Not all of the coefficients in the model need to be subjected to an estimation procedure, some can be set beforehand. This is, in particular, the case for the steady state ratios and similar magnitudes. To begin with some familiar rates or ratios, we put $g^{n}=3 \%$ for the equilibrium growth rate, $h=30 \%$ for the profit share, $\tau=25 \%$ for the tax rate, and $u^{n}=0.90$ for the equilibrium outputcapital ratio (see Franke (2017), for a discussion of the latter). As explained in footnote 7 above, a balanced government budget in the steady state implies $c_{G} / u^{n}=\tau$, which yields $c_{G}=0.225$. Plugging this and the other values into the goods market clearing condition (1.3), the equation can be solved for $c_{o}$ as the only coefficient not yet known; hence $c_{o}=0.0137$.

Let us furthermore set $a^{ \pm}=0.03 \pm 0.03$ for the firms' optimistic and pessimistic capital growth rates. By the specification of $\xi$, this is tantamount to $0.03=1 / \xi$, or $\xi=33.33$. Assume in addition that the firms reconsider their attitude every six months, which gives us $\mu=2.00 .^{21}$ Lastly, the multiplier $1 / D$ in 1.4 plays a crucial role (in Franke (2018a), this is immediately seen, while here, because of the delay in the capital growth rate in (1.6), the effect is less direct). If thus an estimation requires a certain value for $D$, once $\gamma_{u}$ is determined so is $s$, as a residual, or the other way around. Data for a saving rate of the rentiers are not easily available, let alone for their marginal propensity to consume. We thus treat $s$ as one of the parameters to be estimated and, not directly knowing the suitable value of $D$, determine $\gamma_{u}$ residually as half of the investment multiplier, that is, $\gamma_{u}=[\tau+(1-\tau) s h] / 2$ (the estimation then determines $s$ such as to bring 
about the suitable value of $D$ ). The order of magnitude of the resulting saving propensity does not seem unreasonable, so this procedure is justifiable. ${ }^{22}$ In this way there remain 12 parameters to be estimated by our method of simulated moments: $s, \gamma_{k}, \phi_{x}, \phi_{u}, \phi_{k}, \alpha$ for the model's deterministic core and the six stochastic coefficients $\sigma_{u}, \rho_{u}, \sigma_{x}, \rho_{x}, \sigma_{p}, \rho_{p}$.

As mentioned in the Introduction, in this chapter we concentrate on the model's basic merits and demerits in reproducing the real world dynamics regarding output and capital. We are therefore not concerned with distributional issues of the parameter estimates (beginning with their standard errors). In the exploratory stage of our investigations, where as explained above the weights $w_{i}$ in the loss function are given by the reciprocals of the bootstrapped variance of the empirical moments, four moments were found to have particular problems in the matching procedure. These were the autocovariances uu0, uu6, ku9, kk14. To increase their chances, we multiplied their original weights by the factor three. On this basis we decide to settle down on a specific set of numerical parameters values. To approximate the expected values of the moments, the simulations are run over a long horizon of $S=100 \times T=4750$ years. The question as to whether this will be long enough will be taken up further below. Before, with respect to a fixed random seed, we report the resulting point estimates of the parameters in table 1.2 They constitute our reference scenario (RS). Regarding its identification at a still informal level, our explorations with

Table 1.2: Parameters of the reference scenario $R S$.

\begin{tabular}{cccccc}
\hline $100 \times s$ & $\phi_{x}$ & $\phi_{u}$ & $\phi_{k}$ & $\gamma_{k}$ & $\alpha$ \\
\hline 36.09 & 1.153 & 0.000 & 5.366 & 0.569 & 1.246 \\
& & & & & \\
$100 \times \sigma_{x}$ & $\rho_{x}$ & $100 \times \sigma_{p}$ & $\rho_{p}$ & $100 \times \sigma_{u}$ & $\rho_{u}$ \\
\hline 0.415 & 0.178 & 0.208 & 0.338 & 0.112 & 0.880 \\
\hline
\end{tabular}

the numerical minimization algorithm provided no strong evidence that there may be a connected set of some parameters that all yield practically the same minimal value of the loss function. ${ }^{23}$ The only exception is the coefficient $\phi_{u}$, where positive values do not seem to yield a noteworthy improvement. It was therefore fixed at zero straightaway, so that in the rest of the analysis we are concerned with 11 free parameters.

It may not be concealed that there are no persistent cycles in the deterministic core of the reference scenario. Hence, although we started out from an approach that models the upturns and downturns in the business cycle as self-sustaining phenomena, this view is, in a strict sense, not supported by the data. On the other hand, convergence toward the steady state takes place in a cyclical manner. It could thus be said that cyclical behaviour is nevertheless at the heart of our model, but in order to become persistent with all the desired quantitative details, and without stretching the present framework too far, it 
complementarily needs the assistance of some random processes. If the latter are seen as short-cuts of additional mechanisms that, for simplicity, are not explicitly incorporated in the model, the idea of endogenous business cycles is not necessarily rejected. Generally it appears that the debate about whether or not business cycles are in the end generated endogenously is a somewhat futile undertaking. It helps classify specific models, but it is not something that allows a final and universal conclusion. ${ }^{24}$

The profiles of the simulated autocovariance moments to which the chosen parameters give rise are plotted in figure 1.3. It is seen that all of the selected moments (and in fact nearly all of the ACVs up to lag 20) lie inside their confidence interval. This equally holds true for the two raggedness moments. More details characterizing the degree of matching for the single moments will be considered in subsection 1.3.5. It should, however, be checked whether the
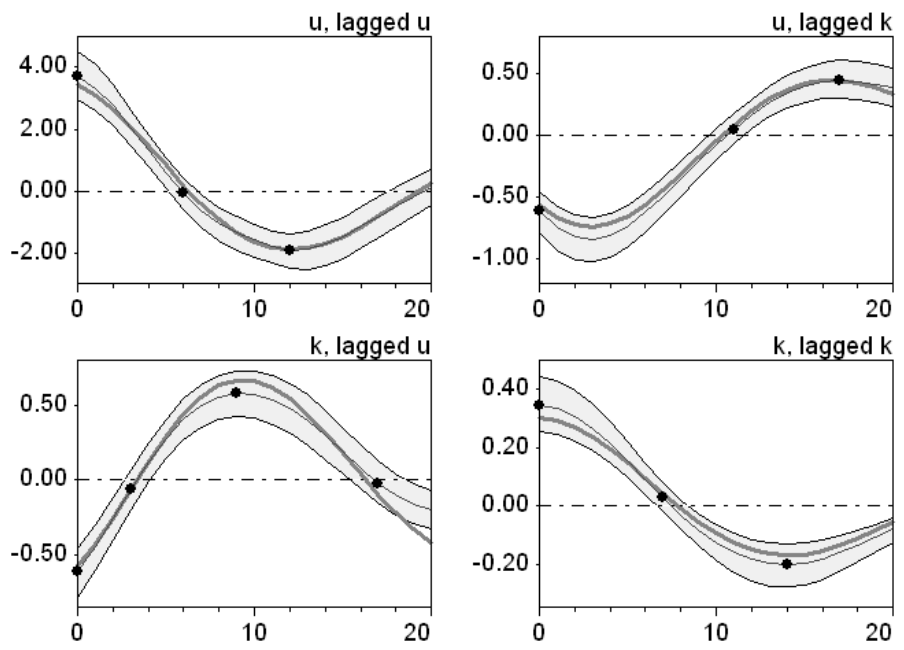

Figure 1.3: Profile of the autocovariances in the reference scenario $R S$ ( $S=4750$ years).

good match exhibited in figure 1.3 is sufficiently representative. This amounts to the question whether, given the parameter values in table 1.2 the moments in the diagram are a good enough approximation to the model's expected moments. The problem is that random number sequences in the simulations that are different from those in the estimation will, of course, yield different moments, however long the simulation period may be.

Therefore, to assess the general quality of the approximation, the moments from a large number of simulations over the same horizon $S=4750$ years have to be computed. On the whole, a total of 5000 runs were conducted, and for each of the single moments we counted the number of times they happened to be in 
their empirical confidence interval. It turned out in this experiment that all of the moments were in all of the runs contained in their intervals except one. This was the covariance ku9, which showed a coverage ratio of 88.5 per cent. In all of these violations the simulated covariance was too high, that is, it exceeded the upper bound of its confidence interval. This information also gives us a first moment diagnostic and reveals a certain shortcoming of the model, although it may not be considered too serious.

\subsubsection{Performance of the model under small sample variability}

It is obvious that the deviations of the simulated from the empirical moments will be generally much wider when the model is only run over the short horizon of $T=47.50$ years. To get an impression of how serious this variability may be, we would like to contrast it with the variability in the real-world data generating process. As such a statistic is not directly available, we proxy it by the variability of the bootstrapped moments from the empirical sample. The information about the moment deviations can be condensed in a single number by computing the corresponding values of the loss function. That is, on the one hand we have a collection of losses $\left\{J^{e m p, b}\right\}_{b=1}^{B}$ for the bootstrapped moments $\left\{m^{e m p, b}\right\}_{b=1}^{B}$ from above, and on the other hand a collection of losses $\left\{J^{M C, c}\right\}_{c=1}^{C}$ for the model's moments $\left\{m_{i}^{c}(\theta ; T)\right\}_{c=1}^{C}$ from a large number $C$ of Monte Carlo simulations ( $\theta$ being the estimated parameter vector). Concretely, $B=C=5000$ will be large enough. The resulting two distributions are plotted in figure 1.4

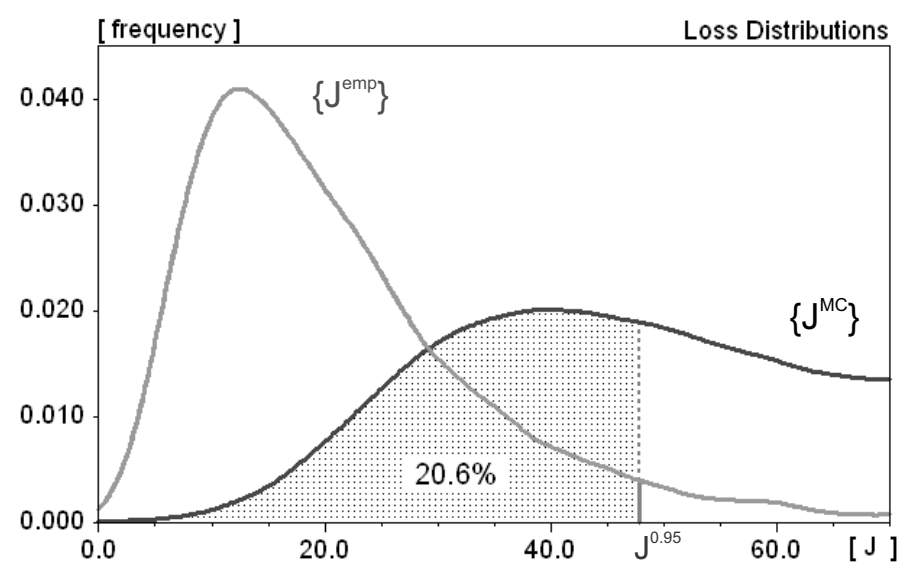

Figure 1.4: Distribution of the bootstrapped empirical losses, $\left\{J^{e m p, b}\right\}$, and the simulated losses of the model under $R S$ with $S=T,\left\{J^{M C, c}\right\}-J_{0.95}$.

The $95 \%$ quantile of $\left\{J^{e m p, b}\right\}$ is $J_{0.95}=47.849$. A sample from a data generating process (DGP) other than that of the real world, which should therefore be 
rejected, would at the familiar $5 \%$ significance level be only rejected as being incompatible with the data if it yields a loss $J$ exceeding this benchmark. Conversely, this cannot be safely done if $J<J_{0.95}$. The DGP as a whole would be rejected if the chance for the latter to happen is less than 5 per cent.

In fact, the probability of a DGP to produce a loss $J$ less than $J_{0.95}$ can be viewed as corresponding to the concept of a $p$-value. Of course, our (and any) model is definitely different from the real-world DGP and thus 'false'. Hence a decision not to reject a DGP on the basis of an observation $J<J_{0.95}$ would be a wrong decision. The $p$-value in our context is the probability of making such an error, i.e., of mistakenly not rejecting a model that is false. ${ }^{25}$ Following the conventional econometric textbook practice it could be said that a model can be reliably rejected if this $p$-value is less than 5 per cent. Figure 1.4, however, shows that the chance of not rejecting our model is as high as 20.6 per cent. In short, regarding its ability to match the empirical moments our model exhibits a statistic of $p=20.6 \%$. In this sense, and on the basis of the chosen moments, there are no sufficient doubts to reject the model as contradicting the data. Therefore, in this sense, it is a fairly good model. ${ }^{26}$

It may be noted that if one agrees on the same set of moments, such a $p$-value can be easily computed for any other model with utilization and a capital ratio among its variables. We have thus a concept at hand that allows us an informed judgement of the overall performance of a model. It opens up the perspective of a model contest in the future that can take the problem of small sample variability into account. In this respect the present model with its $p=20.6 \%$ sets a standard that may serve as a challenge. ${ }^{27}$

\subsubsection{Moment diagnostics}

So far, we have tried an overall assessment of the model's goodness-of-fit. As far as individual moments are concerned, in the Monte Carlo simulations over the long horizon $S=4750$ years in Section 1.3 .3 only the coverage ratio of $88.5 \%$ for moment ku9 has been mentioned, versus the $100 \%$ ratios for all other moments. However, this information need not necessarily mean that ku9 is the hardest moment to match. For example, in most cases the ku9 moments might be close to the empirical value, while otherwise they miss the confidence interval by a small margin only. By contrast, some of the other moments may always be inside the intervals but close to their boundaries. Although this is a somewhat artificial construction, in the following we nevertheless attempt a quantitatively more detailed diagnosis for the 14 moments.

To this end we introduce for a moment $i$ the definition of a moment missing rate $\mathrm{MMR}_{i}$. It measures the distance of a moment $m_{i}$ from its empirical counterpart $m_{i}^{e m p}$ in per cent of the upper or, respectively, lower bound of the confidence interval, depending on whether $m_{i}$ exceeds or falls short of $m_{i}^{e m p}$. Thus, a zero value signifies a perfect match $m_{i}=m_{i}^{e m p}$ and values less (greater) than $100 \%$ indicate that the moment is inside (outside) its confidence interval; such that the higher the rate the worse the match. 
Table (1.3) shows the quantiles of individual moment missing rates from the model's RS simulations. $\mathrm{MMR}_{i}$ in column 2 and 4 , and divided by their average across $i=1, \ldots, 14$ in column 3,5,6. Random seed $\widetilde{c}$ in the simulations over $T=47.5$ years establishes $J^{M C, \widetilde{c}}=20.6 \%$ quantile of $\left\{J^{M C, c}\right\}_{c=1}^{C}$.

Table 1.3: Quantiles from the model's RS simulations.

\begin{tabular}{|c|c|c|c|c|c|}
\hline \multirow{4}{*}{$\begin{array}{r}\text { moment } \\
\mathrm{uu0}\end{array}$} & \multicolumn{5}{|c|}{$\mathrm{MMR}_{i}$, absolute and relative } \\
\hline & \multicolumn{2}{|c|}{ median } & \multicolumn{2}{|c|}{$20.6 \%$ quantile } & \multirow{3}{*}{$\begin{array}{r}\text { rel. } \\
m_{i}^{\tilde{c}}(\theta ; T) \\
1.64\end{array}$} \\
\hline & \multicolumn{2}{|c|}{$m_{i}(\theta ; S) m_{i}(\theta ; S)$} & \multicolumn{2}{|c|}{$m_{i}(\theta ; T) m_{i}(\theta ; T)$} & \\
\hline & 21.5 & 0.65 & 43.7 & 1.29 & \\
\hline $\mathrm{kk} 0$ & 36.9 & 1.11 & 39.4 & 1.16 & 0.05 \\
\hline uk0 & 17.2 & 0.52 & 42.7 & 1.26 & 1.06 \\
\hline uu6 & 50.4 & 1.52 & 17.1 & 0.50 & 0.50 \\
\hline $\mathrm{kk} 7$ & 36.0 & 1.08 & 15.3 & 0.45 & 0.56 \\
\hline $\mathrm{uk} 11$ & 24.0 & 0.72 & 30.3 & 0.89 & 0.24 \\
\hline ku3 & 4.3 & 0.13 & 15.0 & 0.44 & 1.25 \\
\hline $\mathrm{ku} 17$ & 54.8 & 1.65 & 26.4 & 0.78 & 2.79 \\
\hline uu12 & 14.2 & 0.43 & 50.5 & 1.49 & 0.67 \\
\hline $\mathrm{kk} 14$ & 33.1 & 1.00 & 41.8 & 1.23 & 0.23 \\
\hline $\mathrm{uk} 17$ & 16.9 & 0.51 & 51.0 & 1.50 & 0.56 \\
\hline ku9 & 71.0 & 2.14 & 53.5 & 1.58 & 2.03 \\
\hline $\mathrm{Ru}$ & 79.3 & 2.28 & 37.6 & 1.11 & 2.10 \\
\hline $\mathrm{Rk}$ & 5.8 & 0.17 & 11.5 & 0.34 & 0.32 \\
\hline
\end{tabular}

Column 2 and 3 in table 1.3 reconsider the simulations over the horizon $S=$ 4750 in Section 1.3.3. Column 2 presents the median values of the moment missing rates of the single summary statistics. For a better overview of their relative performance, column 3 divides these values by the mean value across the 14 moments. Bold figures indicate the best and worst performing cases. It is thus seen that the critical ku9 moment just mentioned exhibits a relatively high $\mathrm{MMR}_{i}$, too. The raggedness $\mathrm{Ru}$ of the utilization rate, however, is similarly inferior. The fact that nevertheless its moment coverage ratio is as high as $100 \%$ is due to its narrower dispersion across the simulation runs. Conversely, regarding a particularly good match, the covariance ku3 and the raggedness Rk of the capital ratio stand out. 
It will be expected that a similar ranking of the single moments prevails when the model is simulated over the empirical sample period $T=47.50$ years. Against the background of the model's $p$-value in the previous subsection, column 4 and 5 report the $20.6 \%$ quantiles of their $\mathrm{MMR}_{i}$ in likewise absolute and percentage terms, respectively. Again, ku3 and Rk are the best performing moments, now together with kk7, while ku9 shows the worst match. On the other hand, the previously (under $S=4750$ ) inferior raggedness moment $\mathrm{Ru}$ is now in a medium range.

It may furthermore be observed that some rates are below average over the horizon $S$ but above average under horizon $T$ : the moment uu12 with its long lag in the ACV profile and, although less dramatically, the utilization variance uu0 and the contemporaneous covariance $\mathrm{uk} 0$. By contrast, uu6 is an example for the opposite relationship. In order not to get bogged down in too many details, we do not pursue the question how sensitive these reversals in the ranking are to the choice of the quantiles in such comparisons.

In a last experiment we illustrate the tremendous variability in the short Monte Carlo simulations over horizon $T$. This is done in column 6 in table 1.3 , which shows the relative moment missing rates of a selected single run. It is based on the random seed $\widetilde{c}$, the loss $J^{M C, \widetilde{c}}$ of which yields the $20.6 \%$ quantile of the 5000 Monte Carlo losses in this class. Again, Rk is among the best matches and ku9 is especially bad, but the $\mathrm{MMR}_{i}$ of some other moments are in quite a different range from the other columns 3 and 5 .

To take home a short and succinct message, it can be said that the crosscovariance ku9 of medium persistence is particularly hard to match, while the short cross-covariance ku3 of the same variables and the raggedness $\mathrm{Rk}$ of the capital ratio display a remarkable good performance. The matching of the other moments is less clear-cut and can also be somewhat ambiguous. In the end, this may be a consequence of the trade-offs in the summarizing loss function.

\subsubsection{The contribution of the shock processes}

Particularly economists primarily working with deterministic models may be somewhat sceptical about the stochastic attachments to the original model and wonder whether all three of them would be really worth including. To inquire into this question, alternative versions of the model are considered where some of the random processes are excluded, i.e. their parameters are fixed at zero. It may in this respect be remarked that if the estimation includes a process, it usually (but not always) also asks for an active autoregressive part, i.e. a strictly positive autocorrelation coefficient $\rho .^{28}$ In this way there are eight different versions to be estimated: the purely deterministic case, the case when all three stochastic processes are present (giving rise to our reference scenario RS), three cases including only one of the stochastic processes, and three cases including two of them. Throughout, we continue to fix the coefficient $\phi_{u}$ in the switching index (1.11) at zero, as in RS. The resulting parameter estimates together with the minimized values of the loss function $J$ are collected in table 1.4. It shows the estimation of alternative versions of the model for the same random seed $\bar{c}$ (figures rounded). In all cases, $\phi_{u}$ is fixed at zero. 
Table 1.4: Estimation of alternative versions of the model.

\begin{tabular}{|c|c|c|c|c|c|c|c|}
\hline & & & Param & ter sets & & & \\
\hline 1 & 2 & 3 & 4 & 5 & 6 & 7 & $\mathbf{R S}$ \\
\hline$s: 0.382$ & 0.405 & 0.359 & 0.170 & 0.389 & 0.363 & 0.341 & 0.361 \\
\hline$\phi_{x}: 1.246$ & 1.152 & 0.2571 & 1.360 & 1.136 & 1.154 & 0.000 & 1.153 \\
\hline$\phi_{k}: 10.99$ & 5.411 & 10.61 & 10.20 & 5.300 & 5.375 & 15.21 & 5.366 \\
\hline$\gamma_{k}: 0.569$ & 0.593 & 0.082 & 0.704 & 0.586 & .569 & 0.000 & 0.569 \\
\hline$\alpha: 2.398$ & 1.074 & 1.178 & 1.570 & 1.109 & 1.245 & 1.278 & 1.246 \\
\hline $100 \times \sigma_{u}: \quad--$ & 0.127 & -- & -- & 0.120 & 0.112 & -- & 0.112 \\
\hline$\rho_{u}:$ & 0.761 & -- & -- & 0.882 & 0.879 & -- & 0.880 \\
\hline $100 \times \sigma_{z}:$ & -- & 8.073 & -- & 1.467 & $--\varepsilon$ & 8.021 & 0.415 \\
\hline$\rho_{z}: \quad--$ & -- & 0.350 & -- & 0.239 & -- & 0.474 & 0.178 \\
\hline $100 \times \sigma_{p}:$ & -- & $--c$ & 0.250 & -- & 0.208 & 0.214 & 0.208 \\
\hline$\rho_{p}: \quad--$ & -- & $--c$ & 0.160 & -- & 0.348 & 0.555 & 0.338 \\
\hline$J: \quad--$ & 19.9 & 19.0 & 63.2 & 19.4 & 15.3 & 15.4 & 15.2 \\
\hline
\end{tabular}

The limited matching capability of the deterministic model was already mentioned before. As it makes no sense to estimate it on all of the moments, we contended ourselves with an exact match of the first four moments uu0, kk0, uk0, uu6 (see table 1.5). Not all of the five remaining parameters $s, \phi_{x}, \phi_{k}$, $\gamma_{k}, \alpha$ are identified then, that is, $\gamma_{k}$ can be exogenously varied within a certain range and suitable combinations of the other four parameters will still achieve a perfect match. For a specific result, we adopt the value of the reference scenario for $\gamma_{k}$. Even a cursory comparison with the other parameter sets in table 1.4 shows that especially the value of $\alpha$ differs considerably from the stochastic versions of the model. In most of the cases this also holds for $\phi_{k}$. Of course, these differences are a general phenomenon that deterministic model builders may keep in mind in comments on their numerical analysis.

Besides reproducing the values of the loss function, table 1.5 details the missing rates for the single moments. Turning to the stochastic versions and considering the parameter sets $2,3,4$, it is seen that each of the random processes is on its own able to achieve a substantial improvement over the autocovariance profiles of the deterministic model in figure 1.2 apart from the obvious fact that such an influence is needed to produce a certain raggedness in the time series. The most rewarding processes for the moment matching are the rather strongly autocorrelated shocks $\eta_{u, t}$ to aggregate demand in eq. (1.16), which lead to parameter set 2 , and the moderately autocorrelated shocks $\eta_{x, t}$ to the switching index in (1.19), which lead to parameter set 3 . Regarding set 4 , the 
relative inferiority of the perturbations $\eta_{p, t}$ to potential demand in (1.21) will not be very surprising, given their limited transmission mechanisms in the model and their limited ability to introduce some noise in the utilization rate. Table 1.5 also reveals that the three processes affect the single moments in quite different ways. This gives us some hope that a 'cooperation' of two of them may be additionally beneficial. Table (1.5) shows the moment missing rates of $m_{i}(\hat{\theta}, T ; \bar{c})$ in the alternative scenarios for the same random seed $\bar{c}$. The hope is

Table 1.5: Moment missing rates of $m_{i}(\hat{\theta}, T ; \bar{c})$.

\begin{tabular}{rrrrrrrrr}
\hline & \multicolumn{7}{c}{ Parameter sets } \\
& 1 & 2 & 3 & 4 & 5 & $\mathbf{6}$ & $\mathbf{7}$ & RS \\
\hline uu0 : & 0.0 & 35.5 & 44.0 & 94.4 & 36.4 & 33.8 & 27.6 & 33.4 \\
kk0 : & 0.0 & 63.5 & 72.3 & 112.2 & 60.4 & 46.8 & 26.8 & 47.2 \\
uk0 : & 0.0 & 47.4 & 5.3 & 40.4 & 51.1 & 21.9 & 43.5 & 23.0 \\
uu6 : & 0.0 & 42.0 & 4.3 & 80.9 & 43.0 & 50.5 & 0.1 & 50.1 \\
kk7 : & -- & 53.5 & 24.9 & 42.0 & 55.6 & 28.3 & 25.6 & 28.5 \\
uk11 : & -- & 19.0 & 39.0 & 67.3 & 25.2 & 29.6 & 63.8 & 29.5 \\
ku3 : & -- & 10.4 & 20.9 & 33.0 & 1.4 & 5.1 & 61.1 & 4.0 \\
ku17: & -- & 66.2 & 41.8 & 12.7 & 58.8 & 55.4 & 30.5 & 55.1 \\
uu12: & -- & 23.9 & 6.1 & 36.4 & 17.1 & 4.8 & 38.6 & 5.5 \\
kk14: & -- & 29.2 & 61.1 & 86.7 & 36.6 & 41.1 & 69.7 & 41.1 \\
uk17: & -- & 50.4 & 12.6 & 0.5 & 42.7 & 2.9 & 38.1 & 2.9 \\
ku9 : & -- & 42.0 & 80.5 & 105.1 & 50.9 & 58.9 & 53.0 & 59.2 \\
Ru : & -- & 119.0 & 30.2 & 240.0 & 100.6 & 84.6 & 22.6 & 83.9 \\
Rk : & -- & 107.7 & 98.1 & 69.9 & 107.2 & 3.1 & 6.4 & 3.8 \\
\hline$J:$ & -- & 19.9 & 19.0 & 63.2 & 19.4 & $\mathbf{1 5 . 3}$ & $\mathbf{1 5 . 4}$ & $\mathbf{1 5 . 2}$ \\
\hline
\end{tabular}

disappointed if, in the absence to the shocks to potential demand, the shocks to aggregate demand and the sentiment index are combined. Actually, the match of parameter set 5 shown in table 1.4 is slightly inferior to that of set 3 , where the aggregate demand shocks are cancelled. In other words, this set only constitutes a local, but not a global minimum of the loss function $J$. The search algorithm was trapped in it because it was initialized with too high a standard deviation $\sigma_{u}$. Other initializations easily lead to other local minima with quite similar values of $J$. 
While the shocks $\eta_{p, t}$ achieve a relatively poor matching performance on their own, combining them with one of the processes for $\eta_{u, t}$ or $\eta_{x, t}$ is a great success. Parameter sets 6 and 7 in table 1.2 show that with $J=15.3$ or 15.4 their minimized loss is only marginally higher than in the reference scenario with all three shock processes. Hence one of these two processes can practically be regarded as redundant. Even more remarkable is a comparison of scenario RS and set 6 : all of the parameters except $\sigma_{x}$ and $\rho_{x}$, of course, are nearly identical, and also the moment missing rates of the two scenarios in table 1.5 are very similar.

It follows that, in the interest of parsimony, we could and even should eliminate the sentiment shock process from the model and present parameter set 6 as our final showcase scenario - at least as far as the selected 14 moments are concerned. An assessment of its general goodness-of-fit can be made along the lines of the analysis in Section 1.3.4 and 1.3.5. where it will be expected that these results are only marginally inferior to the previous reference scenario. We checked this and, in particular, found: (a) the 5000 simulations over the short empirical horizon $T=47.50$ yield a $p$-value of $20.3 \%$, versus the $20.6 \%$ in RS; (b) the 5000 simulations over the long horizon $S=4750$ yield moment coverage ratios of $100 \%$ for all of the moments except $87.6 \%$ for ku9 (versus $88.6 \%$ in RS) and $99.9 \%$ for $\mathrm{Ru}$ (versus $100 \%$ in RS). These statistics lend further support for parameter set 6 as our preferred estimation. Within the chosen framework this set is the best what we can offer the reader to take home.

\subsection{Conclusion}

The purpose of this chapter was twofold: (1) advancing a most elementary business cycle model, and (2) checking how far it can be made compatible with the data. Regarding (1), Kalecki's and Kaldor's old and practically forgotten idea was revived according to which capacities act negatively on investment and so set up a stabilizing mechanism for the economy. Also referring to Harrod, this aspect could be ascribed to his notion of the firms 'long-range capital outlay', in contrast to the familiar effect of utilization on investment that is based on a shorter time horizon. To meet modern standards, these concepts were adapted to the growth context of the Neo-Kaleckian baseline model with a standard Harrodian instability mechanism, which was enriched with an agentbased story about adjustments of a general business climate. Doing this in a most parsimonious way and remaining within a framework of pure quantity adjustments, a macroeconomic model of a new type was put forward. We christened it a Harrod-Kaldor model in order to indicate that it goes back to the roots of business cycle modelling.

Our aim was beyond paying tribute to our patron saints by designing a modern business cycle model in their spirit. We would like to offer our Harrod-Kaldor model as a core for any heterodox small-scale modelling, into which additional elements from the literature may be easily incorporated. For such an ambition, however, the model should not only be theoretically attractive but, which brings us to the second point, it should also be able to approximate the main 
empirical facts about the business cycle. To this end the model was subjected to an estimation procedure. We chose the method of simulated moments in this respect, because it allows a transparent diagnosis of the merits and demerits of a model. It gives us hints along which dimensions the model shows a good performance and where we would ask too much of it.

We discussed that the dimensions considered, i.e., the 14 moments or summary statistics included in the estimation, are fairly demanding in comparison to similar estimation studies in macroeconomics. Nevertheless, the model was shown to exhibit a remarkably good match of the moments. In a graphical representation this is immediately apparent to the naked eye, but we also proposed the concept of a $p$-value to measure the model's goodness-of-fit quantitatively. As a succinct summary, a $p$-value of around $20 \%$ could be presented.

It was additionally worked out that not all of the components of the general model formulation are jointly needed for a good fit. As a final conclusion of our analysis a parsimonious specification was offered that could be a point of departure for future work. While in our simulation experiments we settled down on a fixed set of numerical values for the coefficients in the model, such work can be concerned with a more systematic investigation of identification issues and the uncertainty surrounding the parameter estimates. This would round off this chapter.

On the other hand, future work can extend the present theoretical framework, preferably by taking one moderate step after the other. For example, Goodwinian themes of income distribution dynamics could be introduced, such that the hitherto constant profit share becomes an endogenous dynamic variable. Or the present overly simple assumption about the firms' financing of investment is differentiated. This may bring banks into the picture and offer some scope for Minskyian themes. Certainly, additional elements for this task can be borrowed from already existing models. An advantage of such a stepwise generalization, complementary to considering these models on their own, is that the Harrod-Kaldor model provides a common theoretical basis for the modelling of the quantity adjustments in the real sector, and it provides a basis that is empirically reliable.

\section{Notes}

${ }^{1}$ Besides the so-called supermultiplier, which gained a lot of attention in the last few years, see Hein et al. (2011) for an overview.

${ }^{2}$ This chapter has been written by Reiner Franke.

${ }^{3}$ Reference to Kaldor is usually made to indicate disequilibrium on the goods market. Here, however, it is meant to indicate the more crucial feature of Kaldor's (1940) trade cycle model, which is the negative influence of the capital stock in his investment function. In fact, Franke (2018a) assumes IS equilibrium. A similar investment function to Kaldor's can already be found in Kalecki (1935, p. 331), although he derives it from a somewhat different argument. 
${ }^{4}$ Because of this limited purpose some may say the chapter is not about estimation but (only) about calibration. The distinction between these terms is, however, often inconsistent and not entirely clear Hansen and Heckman (1996) and so we do not intend to participate in such a dispute about words.

${ }^{5}$ Or the profile of some impulse-response functions, which contains similar information.

${ }^{6}$ Certainly, abstraction itself must not be arbitrary but should satisfy certain methodological criteria; see Nikiforos (2018) for a brief discussion with respect to Kaleckian modelling.

${ }^{7}$ Regarding the financing of government spending it may be noted that a balanced budget in the steady state is guaranteed by the equality of taxes and expenditures, $\tau=\tilde{c}_{G}=c_{G} / u^{n}$, whereas in disequilibrium the government runs a deficit or a surplus. We may think of debt financing, then. Strictly speaking, this would require us to consider interest payments and introduce government debt as an additional dynamic variable (which in turn would require us to extend the discussion of the concept of long-run equilibrium, as sketched in Franke, 2017). To avoid these complications it could provisionally be assumed that the corresponding financial flows exclusively go to or come from foreign countries outside the model.

${ }^{8}$ The latter can be more directly seen from the relationship $g^{i}=[\tau+(1-$ $\tau) s h] u(a, k)-c_{o}-c_{G}$, which is equivalent to 1.5 (solve 1.3 for $g^{i}$ to see this).

${ }^{9}$ This certainly concerns a mathematical stability analysis. Another aspect is that the transition time until such a system comes close to an attractor may be quite long if the initial time path is not appropriately chosen.

${ }^{10}$ An equation like 1.6 is not uncommon in the literature. Observe, however, that in these models it is usually the actual capital growth $q$ that is entering the IS equation and not a function like $g^{i}$, as it is the case in 1.3 .

${ }^{11}$ At least psychologically it would be more reasonable to grant the firms an additional option of moderate growth expectations. Though in a different economic context (Goodwinian income distribution cycles), Franke and Westerhoff (2019) show that this extension easily opens up the possibility of a stable limit cycle to coexist with a stable point equilibrium (or even two). If we focus on the former, such a model should offer a similar perspective to the present binary choice framework. With respect to estimation prospects might be even somewhat better then, a guess that might be worth investigating.

12 Regarding the inflation expectations in the Michigan Survey, there is indeed some evidence for less frequent adjustments. Thus, only around a quarter of the participants update their forecasts in every quarter (Carroll, 2003); or only ten per cent of the forecasts reflect regular information updating (Pfajfar and Santoro 2010).

13 The values of the hyperbolic tangent $\tanh (\cdot)$ are contained between -1 and +1 , it is everywhere strictly increasing, $\tanh (0)=0$ with derivative $\tanh ^{\prime}(0)=1$, and $\tanh (z) \rightarrow \pm 1$ as $z \rightarrow \pm \infty$. An immediate consequence of these properties is that, provided the switching index does not explode over time, the boundaries $x= \pm 1$ are repelling; because $\tanh (z)-x<0(>0)$ for all $x$ close enough to +1 (to -1 , respectively). When $x$ feeds back on the rest of the model, this is a very convenient feature to contain possibly diverging fluctuations.

14 This is virtually the same as simulating the model a great number of times over effectively $T$ periods and taking the mean value of these moments. Regarding the deterministic version of the system, it has been checked that the properties of the 
original model in Franke (2018a) carry over, that is, over a wider range of parameters it converges to a unique limit cycle.

${ }^{15}$ Strictly speaking, the mathematically correct notation would be $R\left(\left\{x_{t}\right\}_{t=1}^{N}\right)$. The case of a zero denominator in 1.29 has never occurred in our applications, so it is neglected in the definition.

${ }^{16}$ The output series is taken from the database fmdata.dat in the zip file fmfp.zip that is provided by Ray Fair on his homepage for working with his macroeconometric model, on http://fairmodel.econ.yale.edu/fp/fp.htm This is a huge plain text file (last accessed December 2015) from which the single time series have to be extracted for further use. Each of them is identified by an acronym. They are explained in Appendix A.4, Table A.2. on pp. 190ff, of the book Estimating How The Macroeconomy Works by R.C. Fair, January 2004, which can be downloaded from http://fairmodel.econ.yale.edu/rayfair/pdf/2003APUB.pdf. The construction of the capital stock series from investment data (as an alternative to the series offered by Fair and Parke) is described in Franke (2017).

${ }^{17}$ Note: The raggedness statistics are computed over the estimation period 1960:q12007:q2. The shaded areas indicate the periods between peaks and troughs as they are classified by the NBER.

18 The effect of the two subperiods on our detrended level variables $u$ and $k$ is less serious. Nevertheless, the model may later be separately re-estimated on these two episodes.

${ }^{19}$ For example, we considered the variance of the output growth rate, for which an almost perfect match was obtained if the model already performed well in the other moments. Matching its first autocorrelation, however, was systematically asking too much given the model's elementary shock processes.

${ }^{20}$ Note: Lags are in quarters and the dots indicate the moments selected for the estimations. The thin solid (red) lines are the profiles from the estimation of the deterministic model (see below).

${ }^{21}$ Hence these firms are more responsive than the subjects mentioned in footnote 12 Besides, $\mu$ is not a very critical parameter. It was checked that suitable adjustments of, in particular, $\phi_{x}, \phi_{u}, \phi_{k}$ after a change in $\mu$ could produce a nearly identical match.

${ }^{22}$ If one has another idea of a reasonable value for $s$ or $\gamma_{u}$, one can take the values of our reference scenario, compute the corresponding $D$, and then vary $s$ and $\gamma_{u}$ such that the value of $D$ is maintained.

23 The Nelder-Mead simplex search algorithm was sufficient for our purpose (see Press et al., 1986, pp. 289-293). On the one hand, it requires less effort than stochastic approaches like simulated annealing. On the other hand, it has broader scope than gradient methods to escape from small local valleys, especially if it is restarted with a larger initial simplex after finding a first minimum.

${ }^{24}$ However, assigning an important role to random processes does not necessarily mean that the incessant cycles are caused by fateful exogenous forces that have to be accepted like yearly natural disasters.

${ }^{25}$ Econometrically, a $p$-value is the probability of mistakenly rejecting a null hypothesis that is true. In the present case the null is the statement that the model is false, a statement that (although many econometricians do not like this mode of expression) is trivially true.

26 The description of this concept of a $p$-value is a bit cumbersome because throughout we painstakingly avoided the phrase that a model may be 'accepted'. 
${ }^{27}$ Of course, a model with a worse performance than another model, i.e. with a lower $p$-value, may nevertheless be 'preferred' on the basis of other reasons.

${ }^{28}$ In some cases this is only found out when the search algorithm is initialized with a moderately high $\rho$; otherwise it may be trapped in an inferior local minimum of the loss function. 
schel, Reiner Franke, Ricardo Araujo, Matthieu Charpe, Christian R. Proaño, and Andreas Szczutkowski - 9781789908008 Downloaded from PubFactory at 04/26/2023 05:01:36AM 